\title{
The Maximum Hamilton Path Problem with Parameterized Triangle Inequality
}

\author{
Weidong Li, Jianping Li ${ }^{*}$, Zefeng Qiao, Honglin Ding \\ ${ }^{1}$ Department of Atmospheric Science, Yunnan University, Kunming, PR China \\ ${ }^{2}$ Department of Mathematics, Yunnan University, Kunming, PR China \\ Email: *jianping@ynu.edu.cn
}

Received 2012

\begin{abstract}
Given a complete graph with edge-weights satisfying parameterized triangle inequality, we consider the maximumHamilton path problem and design some approximation algorithms.
\end{abstract}

Keywords: Maximum Traveling Salesman Problem; Parameterized Triangle Inequality; Approximation Algorithm

\section{Introduction}

Routing design problems are of a major importance incomputer communications and combinatorial optimization. The traveling salesman problem (TSP) and related Hamilton path problem play important role in routing design problems. Recently, some novel approximation algorithms and randomized algorithms are designed for these problems.

Let $G=(V, E, w)$ bea complete graph in which the edge weights satisfy $w(u v) \leq \gamma \cdot(w(u x)+w(x v))$ for all distinct nodes $u, x, v \in V$, the maximum Hamilton path problem(MHP) with $\gamma$-parameterized triangle inequality is to find a path with maximum weight that visits each node exactly once.

A cycle cover is a subgraph in which each vertex in $V$ has a degree of exactly 2 . A maximum cycle cover is one with maximum total edge weight which can be computed in $O\left(n^{3}\right)$ time [4]. It is well-known that the weight of the maximum cycle cover is an upper bound on $O P T$, where $O P T$ denotes the weight of an optimal Hamilton path (or tour). All the algorithms mentioned in this paper start by constructing a maximum-weight cycle cover. A subtour in this paper is a subgraph with no non-Hamiltonian cycles or vertices of degree greater than 2. Thus by adding edges to a subtour it can be completed to a tour. In this paper, for asubset $E^{\prime} \subseteq E, w\left(E^{\prime}\right)$ denotes the (expected) total weight of the edges in $E^{\prime}$.

We call that an algorithm is a $\rho$-approximation algorithm for the MHP if it always produces a feasible solution with objective at least $\rho O P T$, where OPT denotes the optimal value.

When $\gamma=1$, Monnot [10] presented a $1 / 2$-ap-

"Corresponding author. proximation algorithm for the MHP with two specified endpoints and a 2/3 -approximation algorithm for the MHP with one specified end point. To the best of knowledge, this is the unique result about MHP.

In this paper, we first present a deterministic approxiMation algorithm with performance ratio $\frac{4 \gamma+1}{6 \gamma}-\frac{1}{2 n \gamma}$ For the MHP without specified endpoint, and a randomIzed algorithm with expected ratio $\frac{3 \gamma+\frac{1}{2}}{4 \gamma}-O\left(\frac{1}{\sqrt[3]{n}}\right)$ by Modifying the algorithm in [7], where $n$ is the number of vertices in $G$. We also present a determinant approximation algorithm with performance ratio $\frac{4 \gamma+1}{6 \gamma}$ for the $\mathrm{M}$ $\mathrm{H}$

with one specified end point, which improves the result in [10].

A closely related problem is the maximum traveling salesman problem (MTSP) with $\gamma$-parameterized triangleine quality which is to find a tour with maximum weight that visits each node exactly once in a complete graph in which the edge weights satisfy $\gamma$-parameterized triangle in equality. Zhang, Yin, and $\mathrm{Li}$ [14] introduced the MTSP with $\gamma$-parameterized triangle inequality for

$\gamma \in\left[\frac{1}{2}, 1\right)$, motivated by the minimum traveling salesman problem with $\gamma$-parameterized triangle inequality in [13]. They firstcompute a maximum-weight cycle cover $\mathcal{C}=\left\{C_{1}, \ldots, C_{l}\right\}$, and then delete the minimum-weight of the edges in $C_{i}$ and extend the remained paths to a 
Hamiltonian cycle. They [14] proved that the performance ratio of their algorithm is $\frac{K \gamma+1-2 \gamma}{K \gamma}$, where $K=\min \left\{\left|C_{i}\right| \mid i=1,2, \ldots, l\right\}$. Since $\left|C_{i}\right| \geq 3$ for the cycle cover of undirected graph, the ratio is at least $\frac{\gamma+1}{3 \gamma}$.

Notice that the MTSP wit $\gamma \mathrm{h}$-parameterized triangle inequality is a special case of the maximum TSP which is first considered by Fisher, Nemhauser and Wolsey [3], where two approximation algorithms are given. In 1984, Serdyukov[12] presented (in Russian) an elegant $\frac{3}{4}$ - approximation algorithm. Afterwards, Has sin and Rubinstein [6] presented a randomized algorithm whose expected approximation ratio is at least $\frac{25(1-\varepsilon)}{33-32 \varepsilon}$, where $\varepsilon$ is an arbitrarily small constant. Chen, Okamoto, and Wang [2] first gave a deterministic approximation algorithm with the ratio better than $\frac{3}{4}$, which is a $\frac{61}{81}$ - approximation and a nontrivial derandomization of the algorithm from [6]. Very recently, Paluch, Mucha, and Madry [11] first presented a fast deterministic $\frac{7}{9}$-approximation algorithm for the maximum TSP, which isthe currently best result.

If $\gamma=1$, the MTSP with $\gamma$-parameterized triangle in equality is exactly the metric maximum TSP. Kostochka and Serdyukov [7] first designed a $\frac{5}{6}$-approximation algorithm for the metric maximum TSP. Hassin and Rubinstein [5]designed a randomized approximation algorithm for themetric maximum TSP whose expected approximation ratio is $\frac{7}{8}-O\left(\frac{1}{\sqrt[3]{n}}\right)$, where $n$ is the number of vertices in the graph. This algorithm had later been derandomized by Chen and Nagoya [1], at a cost of a slightly worse approximation factor of $\frac{7}{8}-O\left(\frac{1}{\sqrt[3]{n}}\right)$. Very recently, Kowalik and Mucha [9] extended the approach of processing local configuration susing so-called loose-ends in [8], and presented a deterministic $\frac{7}{8}$-approximation algorithm for the metric maximum TSP, which is the currently best result.

The paper is divided into four sections. In Section 2, we will present some algorithms for the MHP problem- without specified endpoint. In Section 3, we will presentan algorithms for the MHP problem with one specified end point. The paper is concluded in the last section.

\section{The MHP Problem without Specified Endpoint}

\subsection{Modified Randomized Kostochka \& Ser- dyukov's Algorithm}

In [5], Hassin and Rubinstein gave a randomized algorithm the maximum TSP [7]. We modify it to the MHP problem.

Algorithm A0:

1. Compute a maximum cycle cover

$$
\mathcal{C}=\left\{C_{1}, C_{2}, \ldots, C_{l}\right\} .
$$

Without loss of generality, we assume that $C_{1}$ satisfies $\frac{w\left(C_{1}\right)}{\left|C_{1}\right|} \leq \frac{w\left(C_{i}\right)}{\left|C_{i}\right|}$ for any $i=1,2, \ldots, l-1$.

2. Delete from each cycle a random edge. Let $u_{i}$ and $v_{i}$ be the ends of the path $P_{i}$ that results from $C_{i}$.

3. Give each path a random orientation and form a Hamilton path HP by adding connecting edges between the head of $P_{i}$ and thetail of $P_{i+1}$, $i=1,2, \ldots, l-1$.

Theorem 1. For any $\gamma \geq \frac{1}{2}$, the expected approximaTion ratio of Algorithm A0 for the MHP problem is

$$
\left(\frac{4 \gamma+1}{6 \gamma}-\frac{1}{2 n \gamma}\right) O P T \text {. }
$$

Proof. Clearly,

$$
\begin{aligned}
w(T)= & \sum_{i=1}^{l} w\left(P_{i}\right)+\frac{1}{4} \sum_{i=1}^{l-1}\left(w\left(u_{i}, u_{i+1}\right)+w\left(u_{i}, v_{i+1}\right)\right. \\
& \left.+w\left(v_{i}, u_{i+1}\right)+w\left(v_{i}, v_{i+1}\right)\right) \\
\geq & \sum_{i=1}^{l} w\left(P_{i}\right)+\frac{1}{2 \gamma} \sum_{i=1}^{l} w\left(u_{i}, v_{i}\right)-\frac{1}{2 \gamma} w\left(u_{1}, v_{1}\right) \\
= & \sum_{i=1}^{l} w\left(C_{i}\right)+\left(\frac{1}{2 \gamma}-1\right) \sum_{i=1}^{l} w\left(u_{i}, v_{i}\right)-\frac{1}{2 \gamma} w\left(u_{1}, v_{1}\right) \\
\geq & \left(1+\frac{1}{K}\left(\frac{1}{2 \gamma}-1\right)-\frac{1}{2 n \gamma}\right) w(\mathcal{C}) \\
\geq & \left(\frac{4 \gamma+1}{6 \gamma}-\frac{1}{2 n \gamma}\right) O P T .
\end{aligned}
$$

Here, the first inequality follows from the $\gamma$-parameterized triangle inequality, the second inequality follows from the assumption that $C_{1}$ satisfies $\frac{w\left(C_{1}\right)}{\left|C_{1}\right|} \leq \frac{w\left(C_{i}\right)}{\left|C_{i}\right|}$, and the last inequality follows from $K \geq 3$. This com- 
pletes the proof. It is well-known that the above randomized version of Kostochka \& Serdyukov's algorithm can easily be derandomized by the standard method of conditional expectation.

\subsection{Modified Hassin \& Rubinstein's Algorithm}

Based on Serdyukov's algorithm in [12] and the randomization version of Kostochka \& Serdyukov's algorithm, Hassin and Rubinstein [5] presented a new algorithm for the maximum TSP problem. We modify it to the MHP problem.

Algorithm B0:

1) Compute a maximum-weight cycle cover $\mathcal{C}=\left\{C_{1}, C_{2}, \ldots, C_{l}\right\}$ of $G$.

2) Compute a maximum-weight matching $M$ in $G$.

3) For $i=2, \ldots, s$, identify $e, f \in E \cap C_{i}$ such thatboth $M \cup\{e\}$ and $M \cup\{f\}$ are subtours. Randomly choose $g \in\{e, f\}$ (each with probability 1/2). Set $P_{i}=C_{i}-\{g\}$ and $M=M \cup\{g\}$.

4) Complete $\bigcup P_{i}$ into a tour $T_{1}$ as in Kostochka \& Serdyukov's algơithm [7].

5) Let $S$ be the set of end nodes of paths in $M$. Compute a random perfect matching $M_{S}$ over $S$. Delete an edge from each cycle in $M \cup M_{S}$. Arbitrarily complete $M \cup M_{S}$ into a tour $T_{2}$.

6) Let $T$ be the maximum-weighted tour between $T_{1}$ and $T_{2}$ and $e$ be the lightest edge in $T$, output the Hamilton path $H P=T-\{e\}$.

Theorem 2. For any $\gamma \in\left[\frac{1}{2},+\infty\right)$, the expected weight of the tour $T$ returned by the Hassin \& Rubinstein's algorithm[5] for the maximum TSP with $\gamma$-parameterized triangle inequality satisfies

$$
w(T) \geq\left(\frac{3 \gamma+\frac{1}{2}}{4 \gamma}-O\left(\frac{1}{\sqrt{n}}\right)\right) O P T .
$$

Proof. Denote by $\alpha$ the relative weight in $C$ of the edges that were candidates for deletion. Let $O P T^{\prime}$ denote the value of the maximum-weighted Hamilton cycle. Clearly, $O P T^{\prime} \geq O P T$. Similarly to the proof of Theorem 1 in [5], we obtain

$$
w\left(T_{1}\right) \geq\left(1-\frac{\alpha}{2}+\frac{\alpha}{2} \cdot \frac{1}{2 \gamma}\right) w(\mathcal{C}) \geq \frac{4 \gamma+(1-2 \gamma) \alpha}{4 \gamma} O P T^{\prime} .
$$

Also, we have

$$
\begin{aligned}
& w\left(M_{S}\right) \geq \frac{1-\alpha}{4 \gamma} w(\mathcal{C}) \\
& w\left(M \cup M_{S}\right) \geq\left(\frac{1}{2}+\frac{\alpha}{2}+\frac{1-\alpha}{4 \gamma}\right) w(\mathcal{C})
\end{aligned}
$$

$$
=\frac{1+2 \gamma-(1-2 \gamma) \alpha}{4 \gamma} \mathrm{OPT}^{\prime}
$$

and

$$
w\left(T_{2}\right) \geq \frac{1+2 \gamma-(1-2 \gamma) \alpha}{4 \gamma}\left(1-O\left(\frac{1}{\sqrt[3]{n}}\right)\right) O P T^{\prime} .
$$

Thus,

$$
\begin{aligned}
\mathrm{w}(T) & =\max \left\{\mathrm{w}\left(T_{1}\right), \mathrm{w}\left(T_{2}\right)\right\} \\
& \geq \frac{\mathrm{w}\left(T_{1}\right)+\mathrm{w}\left(T_{2}\right)}{2} \geq\left(\frac{3 \gamma+\frac{1}{2}}{4 \gamma}-O\left(\frac{1}{\sqrt[3]{n}}\right)\right) O P T^{\prime} .
\end{aligned}
$$

As $e$ is the lightest edge in $T$, we have

$$
\begin{aligned}
w(H P) & \geq\left(1-\frac{1}{n}\right) \mathrm{w}(T) \\
& \geq\left(1-\frac{1}{n}\right)\left(\frac{3 \gamma+\frac{1}{2}}{4 \gamma}-O\left(\frac{1}{\sqrt[3]{n}}\right)\right) O P T^{\prime} \\
& \geq\left(\frac{3 \gamma+\frac{1}{2}}{4 \gamma}-O\left(\frac{1}{\sqrt[3]{n}}\right)\right) O P T .
\end{aligned}
$$

Based on the idea of Chen et al. [2] and the propertiesof a folklore partition of the edges of a $2 n$-vertex complete undirected graph into $2 n-1$ perfect matchings, Chen and Nagoya [1] derandomize Hassin \& Rubinstein's algorithm mat a cost of a slightly worse approximation factor of $\frac{7}{8}-O\left(\frac{1}{\sqrt[3]{n}}\right)$. Similarly to [1], we obtain the following theorem.

Theorem 3. For any $\gamma \geq \frac{1}{2}$, there is a deterministic Algorithm for the maximum TSP with $\gamma$-parameterized triangle inequality with approximation ratio of

$$
\frac{3 \gamma+\frac{1}{2}}{4 \gamma}-O\left(\frac{1}{\sqrt[3]{n}}\right)
$$

\section{The Mhp Problem with One Specified Endpoint}

Let $\mathrm{i}$ be the specified endpoint. The MHP problem with one specified endpoint is to find a maximum- weighted Hamilton path starting from s. Our algorithm is based on computing cycle cover containing the special edge $(s, r)$ for each $r \in V-\{s\}$. We find $n-1$ feasible solutions and output the best one. The detailed algo- 
rithms are presents as follows.

Algorithm A1:

1) For each $r \in V-\{s\}$, compute a maximum cycle cover $\mathcal{C}^{r}=\left\{C_{1}^{r}, C_{2}^{r}, \ldots, C_{l_{r}}^{r}\right\}$ containing the edge $(s, r)$. Assume that $C_{1}^{r}$ contains $(s, r)$ and $w(s, r)=0$.

2) Delete a minimum weight edge from each cycle $C_{i}^{r}$, for $i=2, \ldots, l_{r}$, and the edge $(s, r)$ from $C_{1}^{r}$. Let $u_{i}$ and $v_{i}$ bethe ends of the path $P_{i}$ that results from $C_{i}$, where $u_{1}=s$ and $v_{1}=r$.

3) If $l_{r}=2$, construct two Hamilton paths as follows:

$$
\begin{aligned}
& H P_{1}: s P_{1} r u_{2} P_{2} v_{2}, \\
& H P_{2}: s P_{1} r v_{2} P_{2} u_{2} .
\end{aligned}
$$

If $l_{r} \geq 3$, construct four Hamilton paths as follows.

$$
\begin{aligned}
& H P_{1}: s P_{1} r u_{2} P_{2} v_{2} u_{3} P_{3} v_{3} \cdots u_{l_{r}} P_{l_{r}} v_{l_{r}}, \\
& H P_{2}: s P_{1} r v_{2} P_{2} u_{2} v_{3} P_{3} u_{3} \cdots v_{l_{r}} P_{l_{r}} u_{l_{r}} .
\end{aligned}
$$

When $l$ is odd,

$$
\begin{aligned}
& H P_{3}: s P_{1} r v_{2} P_{2} u_{2} u_{3} P_{3} v_{3} \cdots u_{l_{r}} P_{l_{r}} v_{l_{r}}, \\
& H P_{4}: s P_{1} r u_{2} P_{2} v_{2} v_{3} P_{3} u_{3} \cdots v_{l_{r}} P_{l_{r}} u_{l_{r}} .
\end{aligned}
$$

When $l$ is even,

$$
\begin{aligned}
& H P_{3}: s P_{1} r v_{2} P_{2} u_{2} u_{3} P_{3} v_{3} \cdots v_{l_{r}} P_{l_{r}} u_{l_{r}}, \\
& H P_{4}: s P_{1} r u_{2} P_{2} v_{2} v_{3} P_{3} u_{3} \cdots u_{l_{r}} P_{l_{r}} v_{l_{r}} .
\end{aligned}
$$

4) Compute a maximum-weighted Hamilton path $H P^{r}$ in $\left\{H P_{i} \mid i=1,2,3,4\right\}$ where $H P_{3}=H P_{4}=\varnothing$, if $l_{r}=2$.

5. Output a maximum-weighted Hamilton path $H P$ in $\left\{H P^{r} \mid r \in V-\{s\}\right\}$.

Lemma 4. For each $r \in V-\{s\}$, the objective value of $\mathrm{HP}^{r}$ is at least $\frac{4 \gamma+1}{6 \gamma} w\left(\mathcal{C}^{r}\right)$.

Proof. If $l_{r}=2$, we have

$$
\begin{aligned}
& w\left(H P_{1}\right)+w\left(H P_{2}\right) \\
= & 2 w\left(P_{1}\right)+2 w\left(P_{2}\right)+w\left(r, u_{2}\right)+w\left(r, v_{2}\right) \\
\geq & 2 w\left(P_{1}\right)+2 w\left(P_{2}\right)+\frac{1}{\gamma} w\left(u_{2}, v_{2}\right) \\
\geq & 2 w\left(\mathcal{C}^{r}\right)+\left(\frac{1}{\gamma}-2\right) w\left(u_{2}, v_{2}\right) \\
\geq & 2 w\left(\mathcal{C}^{r}\right)+\left(\frac{1}{\gamma}-2\right) \frac{w\left(C_{2}^{r}\right)}{\left|C_{2}^{r}\right|} \\
\geq & \frac{4 \gamma+1}{3 \gamma} w\left(\mathcal{C}^{r}\right) .
\end{aligned}
$$

Thus,

$$
w\left(H P^{r}\right) \geq \frac{w\left(P_{1}\right)+w\left(P_{2}\right)}{2} \geq \frac{4 \gamma+1}{6 \gamma} w\left(\mathcal{C}^{r}\right) .
$$

If $l_{r} \geq 3$, we have

$$
\begin{aligned}
& \sum_{i=1}^{4} w\left(H P_{i}\right) \\
& =4 \sum_{i=1}^{l_{r}} w\left(P_{i}\right)+2 w\left(r, u_{2}\right)+2 w\left(r, v_{2}\right) \\
& +\sum_{i=2}^{l_{r}-1}\left(w\left(u_{i}, u_{i+1}\right)+w\left(u_{i}, v_{i+1}\right)+w\left(v_{i}, u_{i+1}\right)+w\left(v_{i}, v_{i+1}\right)\right) \\
& \geq 4 \sum_{i=1}^{l_{r}} w\left(P_{i}\right)+\frac{2}{\gamma} \sum_{i=2}^{l_{r}} w\left(u_{i}, v_{i}\right) \\
& =4 w\left(\mathcal{C}^{r}\right)+\left(\frac{2}{\gamma}-4\right) \sum_{i=2}^{l_{r}} w\left(u_{i}, v_{i}\right) \\
& \geq \frac{8 \gamma+2}{3 \gamma} w\left(\mathcal{C}^{r}\right),
\end{aligned}
$$
where the second inequality follows from $\gamma \geq \frac{1}{2}$, and
the last inequality follows from $K \geq 3$ and $w(\mathcal{C}) \geq O P T$. Therefore,

$$
w\left(H P^{r}\right) \geq \frac{1}{4} \sum_{i=1}^{4} w\left(H P_{i}\right) \geq \frac{4 \gamma+1}{6 \gamma} w\left(\mathcal{C}^{r}\right) .
$$

This completes the proof.

Theorem 5. The weight of the Hamilton path HP is at least $\frac{4 \gamma+1}{6 \gamma} O P T$.

Proof. Since HP is the maximum-weighted Hamilton pathin $\{H P r \mid r \in V-\{s\}\}$, we have

$$
\begin{aligned}
w(H P) & \geq \max _{r \in V-\{s\}} w\left(H P^{r}\right) \\
& \geq \max _{r \in V-\{s\}} \frac{4 \gamma+1}{6 \gamma} w\left(\mathcal{C}^{r}\right) \\
& \geq \frac{4 \gamma+1}{6 \gamma} O P T,
\end{aligned}
$$

where the second inequality follows from Lemma 4, and thelast inequality follows from the fact $\max _{r \in V-\{s\}} w\left(\mathcal{C}^{r}\right)$ isan obvious upper bound on $O P T$.

\section{Conclusions}

In this paper, we presented some approximation algorithms for two variants of the maximum Hamilton path problem with parameterized triangle inequality. It is interesting to design some better approximation algorithms for these problems.

\section{Acknowledgements}

The work is supported by the National Natural Science Foundation of China [No. 61063011] and the Tianyuan Fund for Mathematics of the National Natural Science 
Foundation of China [No. 11126315].

\section{REFERENCES}

[1] Z.-Z. Chen and T. Nagoya, "Improved Approximation Algorithms for Metric Max TSP,” Journal of Combinatorial Optimization, Vol. 13, No. 4, 2007, pp. 321-336.

doi:10.1007/s10878-006-9023-7

[2] Z.-Z. Chen, Y. Okamoto and L. Wang, "Improved Deterministic Approximation Algorithms for Max TSP," Information Processing Letters, Vol. 95, No. 2, 2005, pp. 333-342. doi:10.1016/j.ipl.2005.03.011

[3] M. L. Fisher, G. L. Nemhauser and L. A. Wolsey, “An Analysis of Approximation for Finding a Maximum Weight Hamiltonian Circuit," Operations Research, Vol. 27, No. 4, 1979, pp. 799-809. doi:10.1287/opre.27.4.799

[4] D. Hartvigsen, "Extensions of Matching Theory," Ph.D. Thesis, Carnegie-Mellon University, Pittsburgh, PA, 1984.

[5] R. Hassin, S. Rubinstein, "A 7/8-approximation Algorithm Formetric Max TSP," Information Processing Letters, Vol. 81, No. 5, 2002, pp. 247-251.

doi:10.1016/S0020-0190(01)00234-4

[6] R. Hassin and S. Rubinstein, "Better Approximations for Max TSP. Information Processing Letters, Vol. 75, No. 4, 2000, pp. 181-186. doi:10.1016/S0020-0190(00)00097-1

[7] A. V. Kostochka and A. I. Serdyukov, "Polynomial Algorithms with the Estimates 3/4 and 5/6 for the Traveling Salesman Problem of the Maximum (in Russian),"
Upravlyaemye Sistemy, Vol. 26, 1985, pp. 55-59.

[8] L. Kowalik and M. Mucha, "35/44-approximation for Asymmetric Maximum TSP with Triangle Inequality," Algorithmica, doi:10.1007/s00453-009-9306-3

[9] L. Kowalik and M. Mucha, "Deterministic 7/8-approximation for the Metric Maximum TSP,” Theoretical Computer Science, Vol. 410, No. 47-49, 2009, pp. 5000-5009.

doi:10.1016/j.tcs.2009.07.051

[10] J. Monnot, "The Maximum Hamiltonian Path Problem with Specified Endpoint(s)," European Journal of Operational Research, Vol. 161, 2005, pp. 721-735. doi:10.1016/j.ejor.2003.09.007

[11] K. Paluch, M. Mucha and A. Madry, “A 7/9 approximation Algorithm for the Maximum Traveling Salesman Problem," Lecture Notes In Computer Science, Vol. 5687, 2009, pp. 298-311. doi:10.1007/978-3-642-03685-9_23

[12] A. I. Serdyukov, "The Traveling Salesman Problem of the Maximum (in Russian),” UpravlyaemyeSistemy, Vol. 25, 1984, pp. 80-86.

[13] T. Zhang, W. Li and J. Li, “An Improved Approximation Algorithm for the ATSP with Parameterized Triangle Inequality," Journal of Algorithms, Vol. 64, 2009, pp. 74-78. doi:10.1016/j.jalgor.2008.10.002

[14] T. Zhang, Y. Yin and J. Li, “An Improved Approximation Algorithm for the Maximum TSP," Theoretical Computer Science, Vol. 411, No. 26-28, 2010, pp. 2537-2541.

doi:10.1016/j.tcs.2010.03.012 